\title{
Esophageal-pleural fistula after intraoperative transoesophageal echocardiography in a patient with enlarged left atrium
}

\author{
Mario Torre ${ }^{1}$, Antonio Longobardi ${ }^{1}$, David Ferrara ${ }^{1}$, David Ferrara ${ }^{1}$, Maria Giovanna \\ Vassallo $^{1}$, Leonardo De Luca ${ }^{2}$, and Enrico Coscioni ${ }^{1}$ \\ ${ }^{1}$ Azienda Ospedaliera Universitaria 'San Giovanni di Dio e Ruggi d'Aragona' \\ ${ }^{2}$ Ospedale Monaldi
}

February 9, 2022

\begin{abstract}
After mitral valve replacement in an 81-year-old woman, there was evidence of an important communication between the esophagus and the right pleura. Diagnosis was confirmed with Oral Gastrografin radiography and esophagoscopy. Thoracic computed tomography scans better indicated fistula location and extension, but also showed the close relationship and the compression of huge left atrium on the oesophagus. Our hypothesis was that the lesion was induced by transoesophageal echocardiography probe in a favourable setting. An enlarged left atrium should be recognized as a risk factor for TEE-induced esophageal perforation, especially in fragile patients, with marked esophagus distortion.
\end{abstract}

Esophageal-pleural fistula after intraoperative transoesophageal echocardiography in a patient with enlarged left atrium

Mario Torre ${ }^{1-2}$, MD, MSc, Antonio Longobardi ${ }^{1}$, MD, David Ferrara ${ }^{1}$, MD, Raffaele Scaffa ${ }^{1}$, MD, Maria Giovanna Vassallo ${ }^{1}$, MD, Leonardo De Luca ${ }^{3}$, MD, Enrico Coscioni ${ }^{1}$, MD.

${ }^{1}$ Division of Cardiac Surgery, A.O.U. San Giovanni e Ruggi d'Aragona, Salerno, Italy

${ }^{2}$ Division of Cardiac Surgery, Università degli Studi Federico II, Naples, Italy

\section{${ }^{3}$ Division of Gastroenterology, Ospedale Monaldi, Naples, Italy}

Corresponding Author: Mario Torre. Cardiac Surgery Division, A.O.U. San Giovanni e Ruggi d'Aragona. Largo Città di Ippocrate - 84131 - Salerno, Italy. Phone: +39089673101; E-mail: mario.torre0@gmail.com

The authors have no conflict of interest to declare.

\section{ABSTRACT}

After mitral valve replacement in an 81-year-old woman, there was evidence of an important communication between the esophagus and the right pleura. Diagnosis was confirmed with Oral Gastrografin radiography and esophagoscopy. Thoracic computed tomography scans better indicated fistula location and extension, but also showed the close relationship and the compression of huge left atrium on the oesophagus. Our hypothesis was that the lesion was induced by transoesophageal echocardiography probe in a favourable setting. An enlarged left atrium should be recognized as a risk factor for TEE-induced esophageal perforation, especially in fragile patients, with marked esophagus distortion.

Keywords: Esophageal fistula, pleural fistula, transoesophageal echocardiography, left atrium, esophageal perforation 
An 81-year-old woman underwent mitral valve replacement with a porcine bioprosthesis because of severe and symptomatic valve regurgitation. Transoesophageal echocardiography (TEE) was used in a conventional intraoperative setting (at baseline, for de-airing and surgical result evaluation) and the probe was inserted without any resistance. On the fourth postoperative day, there was evidence of a yellowish material from the right thoracic drainage, while she was drinking. She was asymptomatic, afebrile, with modest leucocytosis and increase of inflammatory indexes.

Suspecting the existence of an esophageal-pleural fistula, Methylene blue was given orally, and it coloured the chest tube drainage. Parenteral nutrition was

started immediately. Antibiotic prophylaxis was then started and medications were given intravenously.

Oral Gastrografin radiography confirmed a communication between the esophagus and the right pleura (Fig. 1).

Thoracic computed tomography (CT) scans clearly indicated the fistula location (Fig. 2A) and esophagoscopy showed a wide opening $-6 \mathrm{~cm}$ - on the right surface of the distal esophagus (Fig. 2B). No other anomalies were found. We speculated that perforation could be due to ischemia of the esophagus resulting from the combination of TEE probe compression and non-pulsatile flow during a lengthy on pump procedure, in a fragile patient, as it is already well reported in the literature ${ }^{1}$. Moreover, in our patient, CT images clearly showed a giant left atrium - index volume $182 \mathrm{ml} / \mathrm{m}^{2}$ - compressing and displacing the oesophagus, right in the fistulated region. Several studies reported cases of esophagus compression and distortion by enlarged left atrium related to mitral valve disease ${ }^{2}$ : this anatomic feature should be recognized as a risk factor for TEE-related esophageal complications.

Finally, a 15 x $2 \mathrm{~cm}$ autoexpandable esophageal stent was placed endoscopically. No residual communication was observed, and a new CT scan confirmed the good result (Fig. 3).

\section{REFERENCES}

Sainathan S, Andaz S: A systematic review of transesophageal echocardiography-induced esophageal perforation. Echocardiography. 2013 Sep;30(8):977-83.

Kimura A, Mori T, Kihara Y, et al: A case of esophageal perforation after intraoperative transesophageal echocardiography in a patient with a giant left atrium: unexpectedly large distortion of the esophagus revealed on retrospectively constructed three-dimensional imaging. JA Clin Rep. 2019;5(1):21.

\section{FIGURE LEGEND}

Fig. 1 - The asterixis shows the communication between the esophagus and the right pleura at the oral Gastrografin radiography. PL: Right Pleura.

Fig. 2 A - Computed tomography scan displayed pleural-esophageal fistula (indicated by the asterisk) with contrast effusion in the right pleura, the enlarged left atrium and its distortion of the oesophagus, at the level of the lesion. LA: Left Atrium; LV: Left Ventricle; PL: Right Pleura. B - Esophagoscopy showed the level and the extension of the fistula (indicated by the asterisk) on the right surface of the distal oesophagus, for the subsequent stenting procedure. OE: Oesophagus; PL: Right Pleura.

Fig. 3 - Final computed tomography evaluation of stent deployment in the oesophagus (asterisk) with no residual communication. LA: Left Atrium; LV: Left Ventricle. 


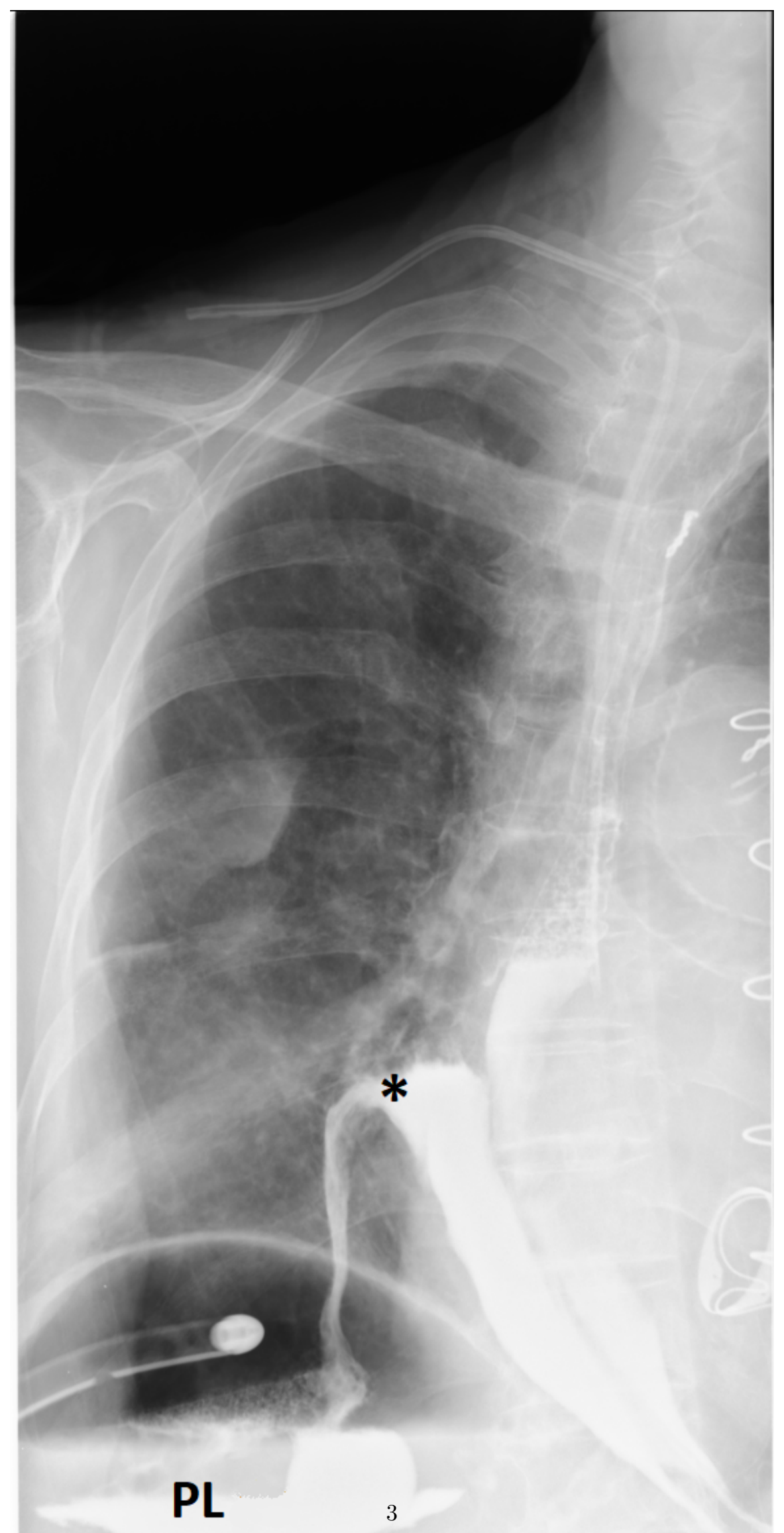



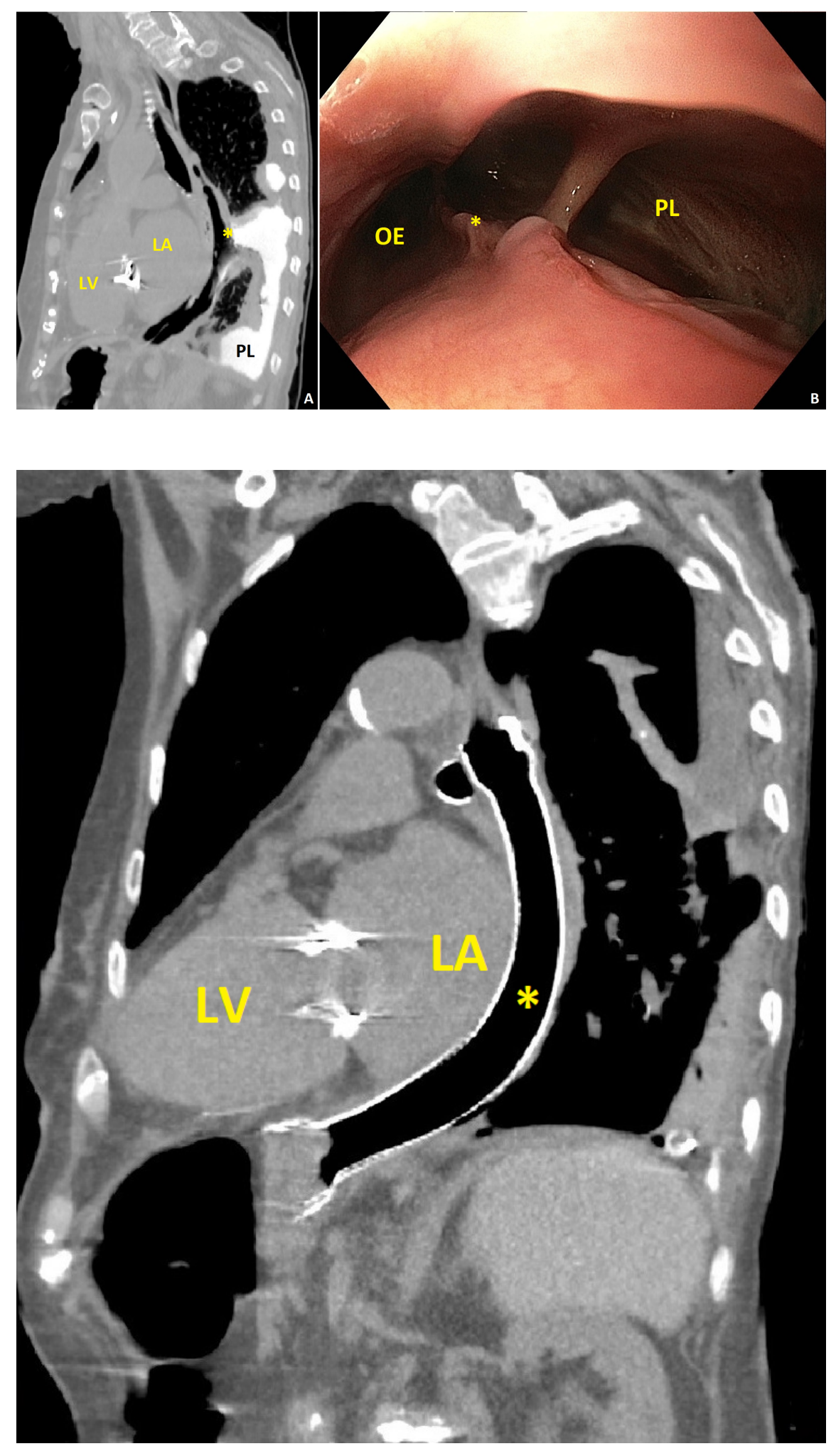